\title{
REPRESENTATION AND APPROXIMATION OF PSEUDODIFFERENTIAL OPERATORS BY SUMS OF GABOR MULTIPLIERS
}

\author{
KARLHEINZ GRÖCHENIG
}

Dedicated to Paul L. Butzer

\begin{abstract}
We investigate a new representation of general operators by means of sums of shifted Gabor multipliers. These representations arise by studying the matrix of an operator with respect to a Gabor frame. Each shifted Gabor multiplier corresponds to a side-diagonal of this matrix. This representation is especially useful for operators whose associated matrix possesses some offdiagonal decay. In this case one can completely characterize the symbol class of the operator by the size of the symbols of the Gabor multipliers. As an application we derive approximation theorems for pseudodifferential operators in the Sjöstrand class.
\end{abstract}

\section{INTRODUCTION}

One of the approaches to understand a given operator is to decompose (= "analyze") the operator into simpler operators and then to find approximation theorems. The meaning of "simple" and "approximation" varies with one's point of view and with the application at hand. In this investigation we take the point of view of time-frequency analysis and consider an operator as simple if it aligns well with Gabor frames. Technically, the simple operators are Gabor multipliers. Given a point $z=(x, \xi) \in \mathbb{R}^{2 d}$ in the time-frequency plane, we denote the associated time-frequency shift acting on a function $f$ by

$$
\pi(z) f(t)=e^{2 \pi i \xi \cdot t} f(t-x), \quad x, \xi, t \in \mathbb{R}^{d} .
$$

Now fix a non-zero function $g \in L^{2}\left(\mathbb{R}^{d}\right)$ (a so-called "window" function) and a lattice $\Lambda=A \mathbb{Z}^{2 d}$ (with an invertible $2 d \times 2 d$-matrix). A Gabor multiplier with the symbol $\mathbf{a}=\left(a_{\lambda}\right)_{\lambda \in \Lambda}$ is defined informally as

$$
M_{\mathbf{a}}^{g, \Lambda} f=\sum_{\lambda \in \Lambda} a_{\lambda}\langle f, \pi(\lambda) g\rangle \pi(\lambda) g .
$$

Gabor multipliers have attracted attention both in engineering and in mathematics $[2,5,9,10,23]$, because they provide an easy and computationally attractive model of time-frequency masking. In (1) the Gabor coefficients $\langle f, \pi(\lambda) g\rangle$ are a

2000 Mathematics Subject Classification. 42C15, 42B15,35S05, 42A16 47G30, $94 \mathrm{~A} 12$.

Key words and phrases. Gabor frame, pseudodifferential operator, time-frequency analysis, Gabor multiplier, approximation theorem, almost diagonalization.

K. G. was supported by the Marie-Curie Excellence Grant MEXT-CT 2004-517154 and in part by the FWF grant SISE S10602. 
measure of the time-frequency content at $\lambda=\left(\lambda_{1}, \lambda_{2}\right) \in \mathbb{R}^{2 d}$, i.e., the amplitude of the frequency $\lambda_{2}$ at time $\lambda_{1}$. The multiplication of the Gabor coefficients with the symbol or mask a amounts to an enhancement or damping of certain time-frequency regions. In this sense, the symbol a can be compared to the transfer function of a discrete time-invariant system usually given by a convolution operator $f \rightarrow f * a$.

Whereas the basic properties (boundedness, mapping properties, Schatten class properties) of Gabor multipliers are well understood [10], it is still open how Gabor multipliers fit into the general picture of (pseudodifferential) operators. How big is the class of Gabor multipliers? How well can a given operator be approximated by a Gabor multiplier or by a sum of Gabor multipliers? Which properties influence and determine the accuracy of an approximation by Gabor multipliers?

To address these questions, we will prove results of the following type.

(1) We show that every operator $A$ from $\mathcal{S}\left(\mathbb{R}^{d}\right)$ to $\mathcal{S}^{\prime}\left(\mathbb{R}^{d}\right)$ can be represented as a sum of shifted Gabor multipliers

$$
A=\sum_{\nu \in \Lambda} \pi(\nu) M_{\mathbf{a}_{\nu}}^{g, \Lambda}
$$

for suitable windows $g$ and symbols sequences $\mathbf{a}_{\nu}$. This decomposition is a new representation of operators comparable to the Schwartz kernel theorem (which we will indeed use to deduce (2) ).

(2) We relate the properties of the symbol of a pseudodifferential operator and the multiplier sequences $\mathbf{a}_{\nu}$. One of the main results is a characterization of the generalized Sjöstrand classes by the size of the multipliers $\mathbf{a}_{\nu}$.

(3) We estimate the error

$$
\left\|A-\sum_{|\nu| \leq N} \pi(\nu) M_{\mathbf{a}_{\nu}}\right\|_{L^{2} \rightarrow L^{2}}
$$

in the operator norm on $L^{2}\left(\mathbb{R}^{d}\right)$ and on other function spaces. In this way we develop an approximation theory for operators. One can draw an analogy to the classical theorems of Jackson for the approximation of a continuous function by trigonometric polynomials, e.g., [3]. Instead of continuous functions we consider bounded operators, and instead of trigonometric polynomials we approximate with finite sums of Gabor multipliers. Our main result is in the spirit of Jackson's theorem: the rate of approximation by sums of Gabor multipliers is directly correlated to the smoothness of the symbol.

(4) Finally we indicate how Gabor multipliers are in the modeling time-varying environments in wireless communication.

A related investigation of Gabor multipliers was carried out by Dörfler and Torresani [5]. They approximate Hilbert-Schmidt operators by Gabor multipliers and sums of Gabor multipliers with different windows and perform a window optimization. Though similar in spirit, the results and methods are completely disjoint. The Hilbert-Schmidt norm of an operator is accessible to explicit calculations, and questions of best approximation and error estimates can be treated by using orthogonal projections. By contrast, there is no formula for the operator norm of an 
operator, and we have to use different ideas. Our results are based on the almost diagonalization of pseudodifferential operators with respect to Gabor frames [13] and the analogy between matrices and operators. In this analogy a Gabor multiplier corresponds to a diagonal matrix, and the representation (2) corresponds to the decomposition of a matrix into the sum of its side-diagonals. The approximation in the operator norm is also motivated by the needs of wireless communications. There the arising operators are perturbations of the identity operator and of convolution operators and they can never be Hilbert-Schmidt operators.

The paper is organized as follows: In Section 2 we recall a minimum of definitions and results from time-frequency analysis, in Section 3 we introduce the main tool for the approximation theory by Gabor multipliers, namely the almost diagonalization of pseudodifferential operators in the Sjöstrand class. Section 4 presents the formal definition and boundedness properties of Gabor multipliers. In Section 5 we derive several versions of the operator representation (2), and in Section 6 we investigate the approximation of operators by Gabor multipliers. We conclude with a brief discussion of Gabor multipliers in wireless communications in Section 7.

\section{Some Time-Frequency Analysis}

Let $z=(x, \xi) \in \mathbb{R}^{d} \times \mathbb{R}^{d}$ be a point in the time-frequency plane, $t \in \mathbb{R}^{d}$. The time-frequency shift $\pi(z)$ is the operator

$$
\pi(z) f(t)=e^{2 \pi i \xi \cdot t} f(t-x)
$$

acting on $L^{2}\left(\mathbb{R}^{d}\right), \mathcal{S}^{\prime}\left(\mathbb{R}^{d}\right)$ and many other spaces. In the following we use [11] as a general reference to time-frequency analysis.

Associated to this set of operators are a signal transform, function spaces, and structured frames; namely the short-time Fourier transform, the modulation spaces, and Gabor frames.

Fix a non-zero window function $g, g \in \mathcal{S}$, say. The short-time Fourier transform of $f$ with respect to the "window" $g$ is defined as

$$
V_{g} f(z)=\langle f, \pi(z) g\rangle=\int_{\mathbb{R}^{d}} f(t) \overline{g(t-x)} e^{2 \pi i \xi \cdot t} d t=(f \cdot g(.-x))(\xi),
$$

whenever the duality $\langle\cdot, \cdot\rangle$, the integral, or the Fourier transform are defined.

Further, we may associate a class of function spaces to the time-frequency shifts and the short-time Fourier transform. Again for fixed non-zero window function $g$, $g \in \mathcal{S}$, say, we define the modulation spaces $M_{m}^{p, q}$ for $1 \leq p, q \leq \infty$ and $m$ a weight function as follows:

$$
f \in M_{m}^{p, q}\left(\mathbb{R}^{d}\right) \Leftrightarrow V_{g} f \in L_{m}^{p, q}
$$

with norm $\|f\|_{M_{m}^{p, q}}=\left\|V_{g} f\right\|_{L_{m}^{p, q}}$. Here $L_{m}^{p, q}$ is the usual mixed-norm space. These spaces are well-defined, there is an extensive theory about modulation spaces. The reader should consult [11, Chps. 11-13] and the references therein, an important source for the history is [6].

Another notion involving the set of time-frequency shifts $\pi(z)$ is the notion of Gabor frames. Fix a lattice $\Lambda \subseteq \mathbb{R}^{2 d}$, i.e., $\Lambda=A \mathbb{Z}^{2 d}$ for an invertible $2 d \times 2 d$ matrix $A$. The collection of time-frequency shifts $\mathcal{G}(g, \Lambda)=\{\pi(\lambda) g: \lambda \in \Lambda\}$ for 
some non-zero $g \in L^{2}\left(\mathbb{R}^{d}\right)$ is called a Gabor system. The set $\mathcal{G}(g, \Lambda)$ is a Gabor frame, if there exist constants $A, B>0$ such that

$$
A\|f\|_{2}^{2} \leq \sum_{\lambda \in \Lambda}|\langle f, \pi(\lambda) g\rangle|^{2} \leq B\|f\|_{2}^{2} \quad \forall f \in L^{2}\left(\mathbb{R}^{d}\right) .
$$

If $A=B=1, \mathcal{G}(g, \Lambda)$ is called a Parseval frame and (3) implies the expansion

$$
f=\sum_{\lambda \in \Lambda}\langle f, \pi(\lambda) g\rangle \pi(\lambda) g \quad \forall f \in L^{2}\left(\mathbb{R}^{d}\right)
$$

with unconditional convergence in $L^{2}\left(\mathbb{R}^{d}\right)$. In contrast to an orthonormal expansion the coefficients in this expansion need not be unique, the choice $\langle f, \pi(\lambda) g\rangle$ is a distinguished, explicit, and convenient choice. The existence problem for Gabor frames is almost completely settled [1]. In what follows, we take the existence of Gabor frames and Parseval Gabor frames for granted.

Characterizations of modulation spaces by Gabor frames. In the following $v$ always denotes a submultiplicative, even weight on $\mathbb{R}^{2 d}$, i.e., $v(x+y) \leq$ $v(x) v(y)$. A weight function $m$ on $\mathbb{R}^{2 d}$ is called $v$-moderate, if $m(x+y) \leq C v(x) m(y)$ for all $x, y \in \mathbb{R}^{2 d}$.

If $\mathcal{G}(g, \Lambda)$ is a Gabor frame and $g \in M_{v}^{1}\left(\mathbb{R}^{d}\right)$, then magnitude of the frame coefficients $\langle f, \pi(\lambda) g\rangle, \lambda \in \Lambda$, characterizes the membership of a function in a particular modulation space $[8,11]$. More precisely, for every $p \in[1, \infty]$ and $v$-moderate weight $m$,

$$
f \in M_{m}^{p, p}\left(\mathbb{R}^{d}\right) \Leftrightarrow\left(\sum_{\lambda \in \Lambda}|\langle f, \pi(\lambda) g\rangle|^{p} m(\lambda)^{p}\right)^{1 / p}<\infty,
$$

and the latter expression is an equivalent norm on $M_{m}^{p, p}$. An analogous characterization holds for rectangular lattices and the mixed modulation spaces $M_{m}^{p, q}$ [8]. For $p \neq q$ and a general lattice $\Lambda$, we have $f \in M_{m}^{p, q}$, if and only if $\sum_{\lambda \in \Lambda}|\langle f, \pi(\lambda) g\rangle| \chi_{\lambda+Q} \in$ $L_{m}^{p, q}\left(\mathbb{R}^{2 d}\right)[7]$.

In particular, we have the following simple characterization of the Schwartz class by means of Gabor frames. Assume that $g \in \mathcal{S}\left(\mathbb{R}^{d}\right)$ and that $\mathcal{G}(g, \Lambda)$ is a frame for $L^{2}\left(\mathbb{R}^{d}\right)$. A function $f \in L^{2}\left(\mathbb{R}^{d}\right)$ belongs to $\mathcal{S}\left(\mathbb{R}^{d}\right)$, if and only if $\langle f, \pi(\lambda) g\rangle, \lambda \in \Lambda$ decays rapidly, i.e.,

$$
|\langle f, \pi(\lambda) g\rangle|=\mathcal{O}\left((1+|\lambda|)^{-s}\right) \quad \text { for all } s \geq 0 .
$$

Pseudodifferential Operators. Given a function (or distribution) $\sigma$ on $\mathbb{R}^{2 d}$, the corresponding pseudodifferential operator with symbol $\sigma$ is defined informally by the integral

$$
\sigma(x, D) f(x)=\int_{\mathbb{R}^{d}} \sigma(x, \xi) \hat{f}(\xi) e^{2 \pi i x \cdot \xi} d \xi
$$

Again the definition of pseudodifferential operators does not necessarily require that the integral is defined, see $[12,16]$ for a rigorous definition. 
REPRESENTATION AND APPROXIMATION OF PSEUDODIFFERENTIAL OPERATORS 5

Symbol Classes. We consider certain modulation spaces as symbol classes. They are generalizations of the Sjöstrand class [18] and may be understood as nonsmooth extensions of the Hörmander class $S_{0,0}^{0}$. Fix a non-zero test function $\Phi$ on $\mathbb{R}^{2 d}$, e.g., the Gaussian $\Phi(z)=e^{-\pi z \cdot z}$ and a submultiplicative weight $v$ on $\mathbb{R}^{2 d}$. Then a symbol $\sigma$ belongs to $M_{v}^{\infty, 1}\left(\mathbb{R}^{2 d}\right)$, if

$$
\int_{\mathbb{R}^{2 d}} \sup _{z \in \mathbb{R}^{2 d}}\left|V_{\Phi} \sigma(z, \zeta)\right| v(\zeta) d \zeta=\|\sigma\|_{M_{v}^{\infty, 1}}<\infty
$$

Likewise, a symbol $\sigma$ belongs to $M_{v}^{\infty, \infty}\left(\mathbb{R}^{2 d}\right)$, if

$$
\sup _{\zeta \in \mathbb{R}^{2 d}} \sup _{z \in \mathbb{R}^{2 d}}\left|V_{\Phi} \sigma(z, \zeta)\right| v(\zeta)=\|\sigma\|_{M_{v}^{\infty, \infty}<\infty}
$$

The generalized Sjöstrand classes are thus certain modulation spaces on $\mathbb{R}^{2 d}$. Further generalizations use the class of solid convolution algebras on $\mathbb{Z}^{2 d}$ to parametrize the extensions of $S_{0,0}^{0}[14]$.

\section{Almost Diagonalization}

We will study the matrix of a pseudodifferential operator $\sigma(x, D)$ with respect to a Gabor frame.

Precisely, let $\mathcal{G}(g, \Lambda)$ be a Gabor system and $\sigma$ a symbol with corresponding pseudodifferential operator $\sigma(x, D)$. Then we consider the matrix $M=M(\sigma)$ with entries

$$
M(\sigma)_{\lambda, \mu}=\langle\sigma(x, D)(\pi(\mu) g), \pi(\lambda) g\rangle \quad \lambda, \mu \in \Lambda .
$$

The main results of $[13,14]$ establish a precise link between pseudodifferential operators in generalized Sjöstrand classes and the associated matrix. In the following $j$ denotes the rotation $j\left(z_{1}, z_{2}\right)=\left(z_{2},-z_{1}\right)$. (It is needed to use the standard modulation operators instead of some symplectic modulations.)

Theorem 1 ( [13]). Assume that $g \in M_{v}^{1}\left(\mathbb{R}^{d}\right)$ and that $\mathcal{G}(g, \Lambda)$ is a Gabor frame. Then $\sigma \in M_{v \circ j^{-1}}^{\infty, 1}$ if and only if there exists $h \in \ell_{v}^{1}(\Lambda)$ such that

$$
\left|M(\sigma)_{\lambda, \mu}\right|=|\langle\sigma(x, D) \pi(\mu) g, \pi(\lambda) g\rangle| \leq h(\lambda-\mu)
$$

for all $\lambda, \mu \in \Lambda$. Furthermore, inf $\|h\|_{\ell_{v}^{1}}$ with the infimum taken over all h satisfying (10) is an equivalent norm on $M_{v \circ j^{-1}}^{\infty, 1}$.

$R E M A R K$ : If $\mathcal{G}(g, \Lambda)$ is a merely a Gabor system with $g \in M_{v \circ j^{-1}}^{1}\left(\mathbb{R}^{d}\right)$ (but not a frame), then the almost diagonalization (10) still holds. However, in general, the converse is not true in general, because the matrix $M(\sigma)$ does not fully describe the operator $\sigma(x, D)$.

A similar theorem holds for the class $M_{v}^{\infty, \infty}$.

Theorem 2 ( [14]). Assume that $g \in M_{v}^{1}\left(\mathbb{R}^{d}\right)$ and that $\mathcal{G}(g, \Lambda)$ is a Gabor frame. Then $\sigma \in M_{v \circ j^{-1}}^{\infty, \infty}$ if and only if there exists

$$
\left|M(\sigma)_{\lambda, \mu}\right|=|\langle\sigma(x, D) \pi(\mu) g, \pi(\lambda) g\rangle| \leq C v(\lambda-\mu)^{-1}
$$

for all $\lambda, \mu \in \Lambda$. 
Thus in the case of $M_{v}^{\infty, \infty}$ one obtains genuine off-diagonal decay of the associated matrix $M(\sigma)$. The standard weights to describe the decay condition are either polynomial weights $v(z)=\langle z\rangle^{s}=\left(1+|z|^{2}\right)^{s / 2}$ or (sub)exponential weights $v(z)=$ $e^{a|z|^{b}}$ for $a>0$ and $0 \leq b \leq 1$.

Other types of decay conditions are studied in [14].

As a special case we mention the Hörmander Class $S_{0,0}^{0}$ consisting of all $C^{\infty}$ functions on $\mathbb{R}^{2 d}$ with bounded derivatives. This symbol class is related to modulation spaces as follows $[14,22]: S_{0,0}^{0}=\bigcap_{s>0} M_{\langle\zeta\rangle^{s}}^{\infty, \infty}$. As a consequence of Theorem 2 we obtain a characterization of $S_{0,0}^{0}$.

Corollary 3. $g \in \mathcal{S}, g \neq 0, \mathcal{G}(g, \Lambda)$ Gabor frame. Then $\sigma \in S_{0,0}^{0}$ if and only if for every $s \geq 0$ there is a constant $C_{s}$ such that

$$
|\langle\sigma(x, D) \pi(\mu) g, \pi(\lambda) g\rangle| \leq C_{s}(1+|\lambda-\mu|)^{-s} \quad \text { for all } \lambda, \mu \in \Lambda .
$$

For completeness we mention that operators with symbols in one of the generalized Sjöstrand classes $M_{v}^{\infty, 1}$ and $M_{v}^{\infty, \infty}$ are closed under composition and that they are bounded on many modulation spaces $[13,14,19]$.

\section{Gabor Multipliers}

Next we introduce a special class of operators, so-called Gabor multipliers. These operators are particularly simple and, in some sense, correspond to diagonal matrices.

Definition 1. Let $\mathcal{G}(g, \Lambda)$ be a Gabor system. Given "symbol sequence" $\mathbf{a}=$ $\left(a_{\lambda}\right)_{\lambda \in \Lambda}$, the Gabor multiplier $M_{\mathbf{a}}$ is defined to be the operator

$$
M_{\mathbf{a}} f=\sum_{\mu \in \Lambda} a_{\mu}\langle f, \pi(\mu) g\rangle \pi(\mu) g .
$$

Clearly, the definition also depends on the Gabor system $\mathcal{G}(g, \Lambda)$. To indicate the dependence of the Gabor multiplier of all parameters, we would have to write $M_{\mathbf{a}}^{g, \Lambda}$. Since our results holds generically for all Gabor systems in a certain class, we prefer to keep the notation simple and omit the reference to $\mathcal{G}(g, \Lambda)$. Likewise one could use two windows $g$ and $\gamma$ and consider Gabor multipliers of the form $M_{\mathbf{a}} f=$ $\sum_{\mu \in \Lambda} a_{\mu}\langle f, \pi(\mu) g\rangle \pi(\mu) \gamma$. Such generalizations cause only notational complications, but do not change any of the results or arguments.

Gabor multipliers have been studied in detail by Feichtinger and Nowak [10]. Gabor multipliers can also be interpreted as time-frequency localization operators with distributional symbols, and boundedness results follow from the theory of localization operators [4].

As a typical boundedness result we state the following one.

Lemma 4. Assume that $g \in M_{v}^{1}\left(\mathbb{R}^{d}\right)$. If $\mathbf{a} \in \ell^{\infty}(\Lambda)$, then $M_{\mathbf{a}}$ is bounded on every modulation space $M_{m}^{p, q}$ for $1 \leq p, q \leq \infty$ and every $v$-moderate weight $m$ with $a$ uniform bound for the operator norm

$$
\left\|M_{\mathbf{a}}\right\|_{M_{m}^{p, q} \rightarrow M_{m}^{p, q}} \leq C_{\Lambda}\|g\|_{M_{v}^{1}}^{2}\|\mathbf{a}\|_{\infty} .
$$


Proof. The decisive property for the proof is the boundedness of the coefficient operator $f \rightarrow(\langle f, \pi(\lambda) g\rangle)_{\lambda \in \Lambda}$ from $M_{m}^{p, q}\left(\mathbb{R}^{d}\right)$ to the sequence space $\ell_{m}^{p, q}(\Lambda)$ and the synthesis operator $\mathbf{c} \rightarrow \sum_{\lambda \in \Lambda} c_{\lambda} \pi(\lambda) g$. See [11, Thms. 12.2.1-4] or [8] (for rectangular lattices). Using these estimates, we find for $p=q$ that

$$
\begin{aligned}
\left\|M_{\mathbf{a}} f\right\|_{M_{m}^{p, p}} & =\left\|\sum_{\mu \in \Lambda} a_{\mu}\langle f, \pi(\mu) g\rangle \pi(\mu) g\right\|_{M_{m}^{p, p}} \\
& \leq C\|g\|_{M_{v}^{1}}\left(\sum_{\mu \in \Lambda}\left|a_{\mu}\langle f, \pi(\mu) g\rangle\right|^{p} m(\mu)^{p}\right)^{1 / p} \\
& \leq C\|g\|_{M_{v}^{1}}\|\mathbf{a}\|_{\infty}\left(\sum|\langle f, \pi(\mu) g\rangle|^{p} m(\mu)^{p}\right)^{1 / p} \\
& \leq\left(C\|g\|_{M_{v}^{1}}\right)^{2}\|\mathbf{a}\|_{\infty}\|f\|_{M_{m}^{p, p}} .
\end{aligned}
$$

For $p \neq q$ and non-separable lattices the proof is identical, once the sequence space is defined correctly. See [7].

\section{Representations of Operators with Gabor Multipliers}

After these preparations let us now explain how and why Gabor multipliers arise in the theory of pseudodifferential operators. Assume that $\mathcal{G}(g, \Lambda)$ is a Parseval frame, so that (41) holds. We expand both $f$ and $A f=\sigma(x, D) f$ with respect to the frame $\mathcal{G}(g, \Lambda)$. Then $f=\sum_{\mu \in \Lambda}\langle f, \pi(\mu) g\rangle \pi(\mu) g$ and $\sigma(x, D) f=$ $\sum_{\lambda \in \Lambda}\langle A f, \pi(\lambda) g\rangle \pi(\lambda) g$. Substituting the expansion of $f$ into the coefficients $\langle A f, \pi(\lambda) g\rangle$, we obtain that

$$
\begin{aligned}
A f & =\sum_{\lambda \in \Lambda}\langle A f, \pi(\lambda) g\rangle \pi(\lambda) g \\
& =\sum_{\lambda \in \Lambda}\left(\sum_{\mu \in \Lambda}\langle A \pi(\mu) g, \pi(\lambda) g\rangle\langle f, \pi(\mu) g\rangle\right) \pi(\lambda) g \quad \lambda=\mu+\nu \\
& =\sum_{\nu \in \Lambda}\left(\sum_{\mu \in \Lambda}\langle A \pi(\mu) g, \pi(\mu+\nu) g\rangle\langle f, \pi(\mu) g\rangle\right) \pi(\mu+\nu) g \\
& =\sum_{\nu \in \Lambda} \pi(\nu)\left(\sum_{\mu \in \Lambda}\langle A \pi(\mu) g, \pi(\mu+\nu) g\rangle e^{2 \pi i \nu_{1} \cdot \mu_{2}}\langle f, \pi(\mu) g\rangle \pi(\mu) g\right)
\end{aligned}
$$

In the transition to the last line we have used the commutation rule for timefrequency shifts $\pi(\mu+\nu)=e^{2 \pi i \nu_{1} \cdot \mu_{2}} \pi(\nu) \pi(\mu)$.

This calculation is known, of course, to every student of linear algebra; it shows how to express a linear operator by its matrix with respect to a "basis", which in our case we take to be a Gabor frame.

Now define the sequence $\mathbf{a}_{\nu}$ by

$$
\mathbf{a}_{\nu}(\mu)=\langle A \pi(\mu) g, \pi(\mu+\nu) g\rangle e^{2 \pi i \nu_{1} \cdot \mu_{2}}
$$


then the expression in (14) in parenthesis is just a Gabor multiplier with symbol $\mathbf{a}_{\nu}$, and we may rewrite (14) as

$$
\sigma(x, D)=\sum_{\nu \in \Lambda} \pi(\nu) M_{\mathbf{a}_{\nu}} .
$$

At least informally, the above identity shows that every pseudodifferential operator is a sum of shifted Gabor multipliers. In this representation each Gabor multiplier corresponds to a side-diagonal of the associated matrix $M(\sigma)$.

To make this argument precise, we offer several versions. We first show that every reasonable operator can be represented as a sum of shifted Gabor multipliers.

Proposition 5. Let $\mathcal{G}(g, \Lambda)$ be a Parseval frame and $g \in \mathcal{S}\left(\mathbb{R}^{d}\right) \backslash\{0\}$.

(i) If the sequences $\mathbf{a}_{\nu}$ satisfy the growth conditions

$$
\left|\mathbf{a}_{\nu}(\mu)\right| \leq C(1+|\mu|+|\nu|)^{N} \quad \text { for } \lambda, \mu \in \Lambda
$$

then $\sum_{\nu \in \Lambda} \pi(\nu) M_{\mathbf{a}_{\nu}}$ defines a continuous operator from $\mathcal{S}\left(\mathbb{R}^{d}\right)$ to $\mathcal{S}^{\prime}\left(\mathbb{R}^{d}\right)$ and the series converges in the weak operator topology.

(ii) Conversely, assume that $A$ is a continuous operator from $\mathcal{S}\left(\mathbb{R}^{d}\right)$ to $\mathcal{S}^{\prime}\left(\mathbb{R}^{d}\right)$ (with the weak ${ }^{*}$-topology). Then $A$ possesses a representation (16) as a sum of shifted Gabor multipliers with symbols $\mathbf{a}_{\nu}$ satisfying the growth estimate

$$
\left|\mathbf{a}_{\nu}(\mu)\right| \leq C(1+|\mu|+|\nu|)^{N}
$$

for constants $C, N \geq 0$ depending only on $g$ and $A$.

Proof. (i) Fix $\nu \in \Lambda$ and consider the Gabor multiplier $M_{\mathbf{a}_{\nu}} f=$ $=\sum_{\mu \in \Lambda} \mathbf{a}_{\nu}(\mu)\langle f, \pi(\mu) g\rangle \pi(\mu) g$. Since by hypothesis $\mathbf{a}_{\nu}$ grows polynomially and $\langle f, \pi(\mu) g\rangle$ decays rapidly by (5), the coefficients $\mathbf{a}_{\nu}(\mu)\langle f, \pi(\mu) g\rangle$ also decay rapidly, whence the sum converges in $\mathcal{S}\left(\mathbb{R}^{d}\right)$ and $M_{\mathbf{a}_{\nu}} f \in \mathcal{S}\left(\mathbb{R}^{d}\right)$. Consequently the partial sums $\sum_{|\nu| \leq M} M_{\mathbf{a}_{\nu}} f$ are in $\mathcal{S}\left(\mathbb{R}^{d}\right)$, and we only need to verify that the partial sums converge in the weak* sense to an element in $\mathcal{S}^{\prime}\left(\mathbb{R}^{d}\right)$. The convergence follows from

$$
\begin{aligned}
\left|\left\langle\sum_{|\nu| \leq M} M_{\mathbf{a}_{\nu}} f, h\right\rangle\right| & \leq \sum_{|\nu| \leq M}\left|\mathbf{a}_{\nu}(\mu)\right||\langle f, \pi(\mu) g\rangle||\langle\pi(\mu) g, h\rangle| \\
& \leq C_{\ell} \sum_{|\nu| \leq M}(1+|\mu|+|\nu|)^{N}(1+|\mu|)^{-\ell}
\end{aligned}
$$

for arbitrary $\ell \geq 0$. Hence the sum converges, or equivalently, the infinite sum $\sum_{\nu \in \Lambda} M_{\mathbf{a}_{\nu}} f$ converges in the weak-* sense.

(ii) Let $A$ be continuous from $\mathcal{S}\left(\mathbb{R}^{d}\right)$ to $\mathcal{S}^{\prime}\left(\mathbb{R}^{d}\right)$. As suggested by informal derivation (14), we choose the symbols $\mathbf{a}_{\nu}$ to be $\mathbf{a}_{\nu}(\mu)=\langle A \pi(\mu) g, \pi(\mu+\nu) g\rangle e^{2 \pi i \nu_{1} \cdot \mu_{2}}$.

By the Schwartz kernel theorem there exists a tempered distribution $k \in \mathcal{S}^{\prime}\left(\mathbb{R}^{2 d}\right)$, such that $\langle A f, h\rangle_{\mathbb{R}^{d}}=\langle k, h \otimes \bar{f}\rangle_{\mathbb{R}^{2 d}}$ for $f, h \in \mathcal{S}\left(\mathbb{R}^{d}\right)$. Since the STFT of a tempered distribution grows at most polynomially, there exists an $N \geq 0$, such that

$$
|\langle k, \pi(\lambda) g \otimes \overline{\pi(\mu) g}\rangle|=\left|V_{g \otimes \bar{g}} k\left(\lambda_{1}, \mu_{1} ; \lambda_{2},-\mu_{2}\right)\right| \leq C(1+|\lambda|+|\mu|)^{N} \quad \forall \lambda, \mu \in \Lambda .
$$


We deduce the claimed growth estimate for the symbols as follows:

$$
\begin{aligned}
\left|\mathbf{a}_{\nu}(\mu)\right| & =\left|\langle A \pi(\mu) g, \pi(\mu+\nu) g\rangle_{\mathbb{R}^{d}}\right| \\
& =\left|\langle k, \pi(\mu+\nu) g \otimes \overline{\pi(\mu) g}\rangle_{\mathbb{R}^{2 d}}\right| \\
& \leq C(1+|\mu|+|\mu+\nu|)^{N} \leq C^{\prime}(1+|\mu|+|\nu|)^{N} .
\end{aligned}
$$

By Step (i) the sum $\sum_{\nu \in \Lambda} \pi(\nu) M_{\mathbf{a}_{\nu}}$ is a continuous operator from $\mathcal{S}\left(\mathbb{R}^{d}\right)$ to $\mathcal{S}^{\prime}\left(\mathbb{R}^{d}\right)$, and by the choice of the multipliers $\mathbf{a}_{\nu}$ this operator must coincide with the given operator $A$.

Our next version of (16) deals with the representation of pseudodifferential operators.

Lemma 6. Assume that $\mathcal{G}(g, \Lambda)$ is a Parseval frame and $g \in M_{v}^{1}\left(\mathbb{R}^{d}\right)$.

(i) If the sequence of symbols $\mathbf{a}_{\nu}$ satisfies the condition

$$
\sum_{\nu \in \Lambda}\left\|\mathbf{a}_{\nu}\right\|_{\infty} v(\nu)<\infty
$$

then the sum of shifted Gabor multipliers (16) converges in the operator norm on $M_{m}^{p, q}$ for every $1 \leq p, q \leq \infty$ and every $v$-moderate weight $m$.

(ii) If $\sigma \in M_{v \circ j^{-1}}^{\infty, 1}\left(\mathbb{R}^{2 \bar{d}}\right)$, then the series (16) converges in the operator norm of $M_{m}^{p, q}$ to $\sigma(x, D)$, and $\sum_{\nu \in \Lambda}\left\|\mathbf{a}_{\nu}\right\|_{\infty} v(\nu) \leq C\|\sigma\|_{M_{v \circ j-1}^{\infty, 1}}$.

Proof. (i) By (12) the operator norm of $M_{\mathbf{a}_{\nu}}$ on $M_{m}^{p, q}$ is bounded by $C\left\|\mathbf{a}_{\nu}\right\|_{\infty}$. Further, the operator norm of a time-frequency shift $\pi(\nu)$ on $M_{m}^{p, q}$ is bounded by $v(\nu)$. Thus, summing over $\nu$, we obtain

$$
\begin{aligned}
\|\sigma(x, D)\|_{M_{m}^{p, q} \rightarrow M_{m}^{p, q}} & \leq \sum_{\nu \in \Lambda}\left\|\pi(\nu) M_{\mathbf{a}_{\nu}}\right\|_{M_{m}^{p, q} \rightarrow M_{m}^{p, q}} \\
& \leq \sum_{\nu \in \Lambda} v(\nu)\left\|M_{\mathbf{a}_{\nu}}\right\|_{M_{m}^{p, q} \rightarrow M_{m}^{p, q}} \\
& \leq \sum_{\nu \in \Lambda}\left\|\mathbf{a}_{\nu}\right\|_{\infty} v(\nu)<\infty .
\end{aligned}
$$

Thus the series of operators (16) converges absolutely in the operator norm on $M_{m}^{p, q}$.

(ii) First note that by Theorem 1 there exists a $h \in \ell^{1}(\Lambda)$ such that $\|h\|_{\ell_{v}^{1}} \leq$ $C\|\sigma\|_{M_{v \circ j^{-1}}^{\infty, 1}}$ and

$$
\left|\mathbf{a}_{\nu}(\mu)\right|=\left|\langle\sigma(x, D) \pi(\mu) g, \pi(\mu+\nu) g\rangle e^{2 \pi i \nu_{1} \cdot \mu_{2}}\right| \leq h(\nu)
$$

By Step (i), (16) converges absolutely in the operator norm on $M_{m}^{p, q}$, and by the choice of symbols the limit coincides with $\sigma(x, D)$. The norm estimate follows from $\sum_{\nu \in \Lambda}\left\|\mathbf{a}_{\nu}\right\|_{\infty} v(\nu) \leq\|h\|_{\ell_{v}^{1}} \leq C\|\sigma\|_{M_{v \circ j-1}^{\infty, 1}}$.

REMARK: By adjusting the conditions in the above proof, many more versions of (16) can be shown to be meaningful. 
By combining the almost diagonalization of Theorems 1 and 2, we now characterize operators with symbols in the generalized Sjöstrand class by means of sums of Gabor multipliers.

Proposition 7. Fix a submultiplicative weight $v$ and a non-zero $g \in M_{v}^{1}\left(\mathbb{R}^{2 d}\right)$ such that $\mathcal{G}(g, \Lambda)$ is a Parseval frame.

(i) A symbol $\sigma$ belongs to $M_{v \circ j^{-1}}^{\infty, 1}\left(\mathbb{R}^{2 d}\right)$, if and only if there exist sequences $\mathbf{a}_{\nu} \in$ $\ell^{\infty}(\Lambda)$, such that

$$
\sigma(x, D)=\sum_{\nu \in \Lambda} \pi(\nu) M_{\mathbf{a}_{\nu}}
$$

and

$$
\sum_{\nu \in \Lambda}\left\|\mathbf{a}_{\nu}\right\|_{\infty} v(\nu)<\infty
$$

Furthermore, the sequence of multipliers $\mathbf{a}_{\nu}$ can be chosen such that $\sum_{\nu \in \Lambda}\left\|\mathbf{a}_{\nu}\right\|_{\infty} v(\nu) \leq$ $C\|\sigma\|_{M_{v}^{\infty, 1}} \leq C^{\prime} \sum_{\nu \in \Lambda}\left\|\mathbf{a}_{\nu}\right\|_{\infty} v(\nu)$.

(ii) Assume in addition that $v^{-1} * v^{-1} \leq C v^{-1}$ ( $v$ is subconvolutive). Then $\sigma \in M_{v \circ j^{-1}}^{\infty, \infty}\left(\mathbb{R}^{2 d}\right)$, if and only if $\sigma(x, D)$ possesses a representation (17) with

$$
\left\|\mathbf{a}_{\nu}\right\|_{\infty} \leq C v(\nu)^{-1} .
$$

Again the multipliers $\mathbf{a}_{\nu}$ can be chosen, such that $\sup _{\nu \in \Lambda}\left\|\mathbf{a}_{\nu}\right\|_{\infty} v(\nu)$ is an equivalent norm on $M_{v \circ j-1}^{\infty, \infty}$.

Proof. The sufficiency was shown in Lemma 6. If $\sigma \in M_{v \circ j^{-1}}^{\infty, 1}$, then both (18) and (17) are satisfied.

Assume conversely that an operator $A: \mathcal{S}\left(\mathbb{R}^{d}\right) \rightarrow \mathcal{S}^{\prime}\left(\mathbb{R}^{d}\right)$ is given as a sum of Gabor multipliers (17) with symbols satisfying (18). Then by the Schwartz kernel theorem $A$ possesses a symbol $\sigma \in \mathcal{S}^{\prime}\left(\mathbb{R}^{2 d}\right)$ and $A=\sigma(x, D)$.

To show that $\sigma$ is in $M_{v}^{\infty, 1}\left(\mathbb{R}^{2 d}\right)$, we estimate the size of entries $M(\sigma)_{\lambda \mu}$ with respect to a reference frame $\mathcal{G}(\varphi, \Lambda)$ and then apply the characterization of Theorem 1 .

Fix a Gabor frame $\mathcal{G}(\varphi, \Lambda)$ with $\varphi \in \mathcal{S}\left(\mathbb{R}^{d}\right) \cap M_{v}^{1}\left(\mathbb{R}^{d}\right)$. Then the matrix entries $M(\sigma)_{\lambda \mu}=\langle\sigma(x, D)(\pi(\mu) \varphi), \pi(\lambda) \varphi\rangle$ are well-defined. Since $\sum_{\nu \in \Lambda} M_{\mathbf{a}_{\nu}}$ converges weakly, we may interchange the brackets $\langle\cdot, \cdot\rangle$ with the summation over $\nu$ and obtain

$$
\begin{aligned}
M(\sigma)_{\lambda \mu} & =\langle\sigma(x, D) \pi(\mu) \varphi, \pi(\lambda) \varphi\rangle \\
& =\sum_{\nu \in \Lambda}\left\langle\pi(\nu) M_{\mathbf{a}_{\nu}} \pi(\mu) \varphi, \pi(\lambda) \varphi\right\rangle \\
& =\sum_{\nu \in \Lambda} \sum_{\kappa \in \Lambda} \mathbf{a}_{\nu}(\kappa)\langle\pi(\mu) \varphi, \pi(\kappa) g\rangle\langle\pi(\nu) \pi(\kappa) g, \pi(\lambda) \varphi\rangle .
\end{aligned}
$$

Since $g \in M_{v}^{1}$ and $\varphi \in \mathcal{S}\left(\mathbb{R}^{d}\right) \cap M_{v}^{1}\left(\mathbb{R}^{d}\right)$, the sequence

$$
h(\lambda)=|\langle g, \pi(\lambda) \varphi\rangle|
$$


belongs to $\ell_{v}^{1}(\Lambda)$ and $\|h\|_{\ell_{v}^{1}} \leq C\|g\|_{M_{v}^{1}}$ (with a constant depending only on $\varphi$ and

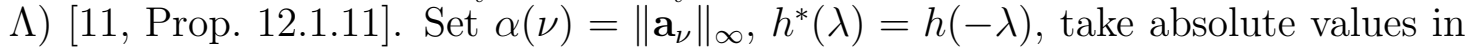
(19), and use the decay of $h$. Then we obtain that

$$
\begin{aligned}
\left|M(\sigma)_{\lambda \mu}\right| & \leq \sum_{\nu \in \Lambda} \sum_{\kappa \in \Lambda}\left\|\mathbf{a}_{\nu}\right\|_{\infty} h(\mu-\kappa) h(\lambda-\nu-\kappa) \\
& =\sum_{\nu \in \Lambda} \alpha(\nu) \sum_{\kappa \in \Lambda} h^{*}(\kappa) h(\lambda-\nu-(\kappa+\mu)) \\
& =\sum_{\nu \in \Lambda} \alpha(\nu)\left(h^{*} * h\right)(\lambda-\mu-\nu) \\
& =\left(\alpha * h^{*} * h\right)(\lambda-\mu) .
\end{aligned}
$$

Since $\alpha \in \ell_{v}^{1}$ by assumption (17) and $h \in \ell_{v}^{1}$ because $g \in M_{v}^{1}$, the matrix $M(\sigma)$ is dominated entrywise by the sequence $\alpha * h^{*} * h \in \ell_{v}^{1}$.

Theorem 1 applies and we conclude that $\sigma \in M_{v \circ j^{-1}}^{\infty, 1}\left(\mathbb{R}^{2 d}\right)$, as claimed. Furthermore,

$$
\begin{aligned}
\|\sigma\|_{M_{v}^{\infty, 1}} & \leq C\left\|\alpha * h^{*} * h\right\|_{\ell_{v}^{1}} \\
& \leq C\|\alpha\|_{\ell_{v}^{1}}\|h\|_{\ell_{v}^{1}}^{2} \\
& \leq C^{\prime}\|\alpha\|_{\ell_{v}^{1}}\|g\|_{M_{v}^{1}}^{2} .
\end{aligned}
$$

The proof for $\sigma \in M_{v \circ j^{-1}}^{\infty, \infty}$ is similar. The only modification occurs in the last part, where we have to use the subconvolutivity of $v$ and the convolution relation $\ell_{v}^{\infty} * \ell_{v}^{\infty} \subseteq \ell_{v}^{\infty}$

\section{Approximation Theorems}

Since every operator $A$ can be represented as an infinite sum of shifted Gabor multipliers, it is natural to truncate the infinite series (17) and approximate $A$ by a finite sum of Gabor multipliers. Compared to a general operator, a finite sum of Gabor multipliers is easy to understand and easy to treat computationally.

For the formulation of the approximation theorems we introduce the error

$$
E_{N}(\sigma):=\left\|\sigma(x, D)-\sum_{|\nu| \leq N} \pi(\nu) M_{\mathbf{a}_{\nu}}\right\|_{M^{p, q} \rightarrow M^{p, q}} .
$$

We take some liberty in the interpretation of the operator norm involved. As we have seen, the spaces we can chose depend mostly on the quality of the window.

Here are some precise approximation theorems.

Theorem 8. Assume that $\mathcal{G}(g, \Lambda)$ is a (Parseval) Gabor frame for $L^{2}\left(\mathbb{R}^{d}\right)$.

(i) If $g \in M^{1}\left(\mathbb{R}^{d}\right)$ and $\sigma \in M^{\infty, 1}$, then $E_{N}(\sigma) \rightarrow 0$ (in the operator norm on $M^{p, q}$ for all $\left.1 \leq p, q \leq \infty\right)$.

(ii) If $g \in \bar{M}_{v}^{1}$ and $\sigma \in M_{v \circ j^{-1}}^{\infty, \infty}$, then

$$
E_{N}(\sigma) \leq C\|\sigma\|_{M_{v \circ j}^{\infty, \infty}}^{\infty, \infty} \sum_{|\nu|>N} v(\nu)^{-1}
$$


(iii) If $g \in M_{v}^{1}$ and $\sigma \in M_{v \circ j^{-1}}^{\infty, 1}$, then

$$
E_{N}(\sigma) \leq C\|\sigma\|_{M_{v \circ j^{-1}}^{\infty, 1}} \sup _{|\nu|>N} v(\nu)^{-1} .
$$

(iv) If $g \in M_{\langle\zeta\rangle^{s}}^{1}$ and $\sigma \in M_{\langle\zeta\rangle^{s}}^{\infty, \infty}$ and $s>2 d$, then

$$
E_{N}(\sigma) \leq C\|\sigma\|_{M_{\langle\zeta\rangle^{s}}^{\infty, \infty}} N^{2 d-s}
$$

(v) If $g \in M_{\langle\zeta\rangle^{s}}^{1}$ and $\sigma \in M_{\langle\zeta\rangle^{s}}^{\infty, 1}$, then

$$
E_{N}(\sigma) \leq C\|\sigma\|_{M_{\langle\zeta\rangle}^{\infty, 1}} N^{-s}
$$

Proof. (i) By Proposition 7 we represent $\sigma(x, D)$ as a sum of Gabor multipliers

$$
\sigma(x, D)=\sum_{\nu \in \Lambda} \pi(\nu) M_{\mathbf{a}_{\nu}},
$$

such that $\sum_{\nu \in \Lambda}\left\|\mathbf{a}_{\nu}\right\|_{\infty}<\infty$. Since the sum converges in the operator norm on $M^{p, q}$, the difference between $\sigma(x, D)$ and its approximation becomes

$$
\sigma(x, D)-\sum_{\nu \in \Lambda} \pi(\nu) M_{\mathbf{a}_{\nu}}=\sum_{|\nu|>N} \pi(\nu) M_{\mathbf{a}_{\nu}} .
$$

Taking operator norms, we obtain the following estimate for the error $E_{N}(\sigma)$ :

$$
\begin{aligned}
E_{N}(\sigma) & =\left\|\sigma(x, D)-\sum_{\nu \in \Lambda} \pi(\nu) M_{\mathbf{a}_{\nu}}\right\|_{M^{p, q} \rightarrow M^{p, q}} \\
& =\left\|\sum_{|\nu|>N} \pi(\nu) M_{\mathbf{a}_{\nu}}\right\|_{M^{p, q} \rightarrow M^{p, q}} \\
& \leq \sum_{|\nu|>N}\left\|M_{\mathbf{a}_{\nu}}\right\|_{M^{p, q} \rightarrow M^{p, q}} \\
& \leq \sum_{|\nu|>N}\left\|\mathbf{a}_{\nu}\right\|_{\infty} .
\end{aligned}
$$

Since $\sigma \in M^{\infty, 1}$ and $\sum_{\nu \in \Lambda}\left\|\mathbf{a}_{\nu}\right\|_{\infty}<\infty$, (21) implies that $E_{N}(\sigma) \rightarrow 0$.

(ii) By Proposition 7 the assumption $\sigma \in M_{v \circ j^{-1}}^{\infty, \infty}$ implies that $\left\|\mathbf{a}_{\nu}\right\|_{\infty} \leq C\|\sigma\|_{M_{v \circ j}^{\infty}, \infty} v(\nu)^{-1}$. Consequently, by (21) the approximation error is at most

$$
E_{N}(\sigma) \leq \sum_{|\nu|>N}\left\|\mathbf{a}_{\nu}\right\|_{\infty} \leq C\|\sigma\|_{M_{v \circ j^{-1}}^{\infty, \infty}} \sum_{|\nu|>N} v(\nu)^{-1} .
$$

If $v(z)=(1+|z|)^{s}$, then $\sum_{|\nu|>N}(1+|\nu|)^{-s} \leq C N^{2 d-s}$, whence assertion (iv).

(iii) Likewise, if $\sigma \in M_{v \circ j^{-1}}^{\infty, 1}$, then by Theorem 1

$$
\sum_{\nu \in \Lambda}\left\|\mathbf{a}_{\nu}\right\|_{\infty} v(\nu) \leq C\|\sigma\|_{M_{v \circ j^{-1}}^{\infty, 1}}<\infty
$$

and thus

$$
E_{N}(\sigma) \leq \sum_{|\nu|>N}\left\|\mathbf{a}_{n}\right\|_{\infty} \leq \sup _{|\nu|>N} v(\nu)^{-1} \sum_{|\nu|>N}\|\mathbf{a}\|_{\infty} v(\nu)
$$


If $v(z)=(1+|z|)^{s}$, then $\sup _{|\nu|>N} v(\nu)^{-1} \leq(1+N)^{-s}$, whence $(\mathrm{v})$.

REMARKS: 1. The error estimates of Theorem 8 yields an estimate for the size of the cut-off parameter $N$ and thus of the number of shifted Gabor multipliers required for a good approximation. For instance, if $\sigma \in M_{\langle\zeta\rangle}^{\infty, 1}\left(\mathbb{R}^{2 d}\right)$ and a tolerance $\epsilon>0$ are given, then the error estimate

$$
E_{N}(\sigma) \leq C\|\sigma\|_{M_{\langle\zeta\rangle}^{\infty, 1}} N^{-s}
$$

implies that

$$
N>\left(\frac{\left(C\|\sigma\|_{M_{\langle\zeta\rangle}^{\infty, 1}}\right.}{\epsilon}\right)^{1 / s} .
$$

It is possible to say more about the dependency of the constant $C$ on $g$ and $\Lambda$ by precise bookkeeping in the results about almost diagonalization of pseudodifferential operators in [13].

2. One may wonder whether a converse of Theorem 8 holds and whether the quality of approximation as expressed by Theorem 8 characterizes the symbol classes. This guess is false, because the operator norm $\|\sigma(x, D)\|_{M^{p, q} \rightarrow M^{p, q}}$ and $\|\sigma\|_{M^{\infty, 1}}$ are not equivalent. Indeed, following an idea of Klotz [17] about approximation algebras of matrices, one can define a new symbol class directly by the approximation properties of Gabor multipliers as follows: We say that $\sigma \in \mathcal{A}^{s}\left(\mathbb{R}^{2 d}\right)$, if

$$
\inf _{\mathbf{a}_{\nu} \in \ell^{\infty}(\Lambda)}\left\|\sigma(x, D)-\sum_{\nu \in \Lambda,|\nu|>N} M_{\mathbf{a}_{\nu}}\right\|_{L^{2} \rightarrow L^{2}} \leq C N^{-s} .
$$

This class of symbols contains $M_{\langle\zeta\rangle^{s}}^{\infty, \infty}$. By comparison with the corresponding matrix algebras one can verify that $M_{\langle\zeta\rangle^{s}}^{\infty, \infty}$ is strictly smaller than $\mathcal{A}^{s}\left(\mathbb{R}^{2 d}\right)$.

\section{Application}

Finally we mention the problem that has motivated the approximation theorems in the previous section. This problem concerns the transmission and decoding of digital information by a variant of (orthogonal) frequency division multiplexing (OFDM).

Here is a very coarse description of this procedure.

1. Given is "digital information" in the form of a finite sequence $\left\{c_{\lambda}: \lambda \in \Lambda\right\}$, where the data are taken from a finite alphabet, usually just $c_{\lambda} \in\{-1,1\}$ or $c_{\lambda} \in\{ \pm 1 \pm i\}$.

2. D/A conversion: To transmit these data, they are converted to an analog signal of the form

$$
f=\sum_{\mu \in \Lambda} c_{\mu} \pi(\mu) g .
$$

for a suitable pulse $g$. Usually the lattice is taken to be a rectangular lattice $\Lambda=$ $\alpha \mathbb{Z} \times \beta \mathbb{Z}$. Clearly, the coefficients must be uniquely determined by $f$. Therefore, and in contrast to the previous discussions, the common assumption in wireless communication is that $\mathcal{G}(g, \Lambda)$ is a Riesz sequence (for a closed subspace) in $L^{2}(\mathbb{R})$. 
3. Transmission of the signal $f$ : The analog signal $f$ is transmitted by a sender. This is a physical process subject to the laws of physics, in particular the wave equation.

4. Distortion of $f$ : During the transmission the signal is distorted by various effects. The most common effects are time lags due to reflection at obstacles and the Doppler effect due to relative motion between transmitter and receiver. Thus the distortion can be modelled by a superposition of time-frequency shifts, and the received signal is of the form

$$
\tilde{f}(t)=\int_{\mathbb{R}} \hat{\sigma}(\eta, u) M_{\eta} T_{-u} f(t) d u d \eta .
$$

Here the weighting factor $\hat{\sigma}$ models the physical details of the transmission, such as the reflectivities of obstacles and the Doppler effect. It is well known that the distortion (22) is precisely the pseudodifferential operator with symbol $\sigma$, thus

$$
\tilde{f}=\sigma(x, D) f=\sum_{\mu \in \Lambda} c_{\mu} \sigma(x, D)(\pi(\mu) g) .
$$

Under the natural assumption of a maximum Doppler shift $\nu_{0}$ and a maximum time lag $\tau_{0}$, the weight function $\hat{\sigma}$ possesses a compact support in $\left[0, \tau_{0}\right] \times\left[-\nu_{0}, \nu_{0}\right]$. If $\hat{\sigma}$ were a bounded function, then the distortion operator $\sigma(x, D)$ would be a HilbertSchmidt operator and could not be invertible. Therefore Strohmer [20] proposed the generalized Sjöstrand class $M_{e^{a|\zeta|}}^{\infty, 1}$ with an exponential weight $v(\zeta)=e^{a|\zeta|}$ as a suitable symbol class to model the distortion of time-varying systems in wireless communications. This class contains the distortion free channel corresponding to the identity operator (with symbol $\sigma \equiv 1$ ) and time-invariant channels corresponding to convolution operators.

5. A/D conversion: At the receiver we decode the original digital information $c_{\lambda}$ from the distorted signal by taking correlations with time-frequency shifts as follows:

$$
y_{\lambda}=\langle\tilde{f}, \pi(\lambda) g\rangle=\sum_{\mu \in \Lambda} c_{\mu} \underbrace{\langle\sigma(x, D)(\pi(\mu) g), \pi(\lambda) g\rangle}=(M(\sigma) \mathbf{c})_{\lambda} .
$$

The output vector $\mathbf{y}=\left(y_{\lambda}\right)_{\lambda \in \Lambda}$ is completely determined by the received signal $\tilde{f}$. With definition (9) the input-output relation can be written as the infinite system of equations

$$
\mathbf{y}=M(\sigma) \mathbf{c}
$$

In wireless communications the matrix $M(\sigma)$ is called the channel matrix.

For (24) to be well posed, the Gabor system $\mathcal{G}(g, \Lambda)$ is assumed to be a Riesz basis for its span $\mathcal{H}_{g, \Lambda}=\operatorname{span} \mathcal{G}(g, \Lambda)$, and the distortion $\sigma(x, D)$ is assumed to be an invertible operator on $L^{2}\left(\mathbb{R}^{d}\right)$. Then the channel matrix $M(\sigma)$ is (boundedly) invertible on $\ell^{2}(\Lambda)$, and the solution to (24) is well defined.

6. Equalization: To solve for the original information $\mathbf{c}$ we need to solve the system of equations (24) and find the solution $\mathbf{c}=M(\sigma)^{-1} \mathbf{y}$.

At this point occurs amazing assumption that is taken for granted by engineers in this field: the channel matrix $M(\sigma)$ is assumed to be a diagonal matrix. 
With this assumption, the distortion $\tilde{f}$ is simply

$$
\begin{aligned}
\tilde{f}=\sigma(x, D) f & =\sum_{\lambda \in \Lambda}\langle\sigma(x, D) \pi(\lambda) g, \pi(\lambda) g\rangle\langle f, \pi(\lambda) g\rangle \pi(\lambda) g \\
& =\sum_{\lambda \in \Lambda} M(\sigma)_{\lambda \lambda}\langle f, \pi(\lambda) g\rangle \pi(\lambda) g .
\end{aligned}
$$

In other words, wireless communications works with the implicit assumption that the distortion operator is a Gabor multiplier with respect to a Riesz sequence $\mathcal{G}(g, \Lambda)$. The main motivation for this assumption seems to be its convenience and simplicity. With this assumption the solution to (24) is simply

$$
c_{\lambda}=M(\sigma)_{\lambda, \lambda}^{-1} y_{\lambda} .
$$

One can show that the channel matrix with respect to a Gabor frame can never be a diagonal matrix. However, if $\sigma \in M_{v}^{\infty, 1}$ with exponential weight $v$, then the channel matrix is almost diagonal with exponential decay. Theorem 8 then guarantees that the distortion operator $\sigma(x, D)$ is approximated extremely well by a finite number of shifted Gabor multipliers, or equivalently, $M(\sigma)$ is approximated extremely well by a banded matrix with few bands. In this case one may hope to improve the accuracy of inversion of (24) by using a banded approximation of $M(\sigma)$ instead of the main diagonal only. In other words, we use an approximation of $\sigma(x, D)$ by a finite sum of shifted Gabor multipliers.

This idea has been tested and implemented in [15,21]. Combined with other tricks, the approximation of the distortion operator by a sum of Gabor multipliers contributed significantly to a low complexity equalizer for time-varying systems. This equalizer performs best in strongly time-varying environments.

\section{REFERENCES}

[1] B. Bekka. Square integrable representations, von Neumann algebras and an application to Gabor analysis. J. Fourier Anal. Appl., 10(4):325-349, 2004.

[2] J. J. Benedetto and G. E. Pfander. Frame expansions for Gabor multipliers. Appl. Comput. Harmon. Anal., 20(1):26-40, 2006.

[3] P. L. Butzer and R. J. Nessel. Fourier analysis and approximation. Academic Press, New York, 1971. Volume 1: One-dimensional theory, Pure and Applied Mathematics, Vol. 40.

[4] E. Cordero and K. Gröchenig. Time-frequency analysis of localization operators. J. Funct. Anal., 205(1):107-131, 2003.

[5] M. Dörfler and B. Torresani. Representation of operators in the time-frequency domain and generalized Gabor multipliers. J. Fourier Anal. Appl., 2009, to appear.

[6] H. G. Feichtinger. Modulation spaces: looking back and ahead. Sampl. Theory Signal Image Process., 5(2):109-140, 2006.

[7] H. G. Feichtinger and K. Gröchenig. Banach spaces related to integrable group representations and their atomic decompositions. I. J. Functional Anal., 86(2):307-340, 1989.

[8] H. G. Feichtinger and K. Gröchenig. Gabor frames and time-frequency analysis of distributions. J. Functional Anal., 146(2):464-495, 1997.

[9] H. G. Feichtinger, M. Hampejs, and G. Kracher. Approximation of matrices by Gabor multipliers. IEEE Signal Proc. Letters, 11(11):883- 886, November 2004.

[10] H. G. Feichtinger and K. Nowak. A first survey of Gabor multipliers. In Advances in Gabor analysis, Appl. Numer. Harmon. Anal., pages 99-128. Birkhäuser Boston, Boston, MA, 2003. 
[11] K. Gröchenig. Foundations of time-frequency analysis. Birkhäuser Boston Inc., Boston, MA, 2001.

[12] K. Gröchenig. A pedestrian's approach to pseudodifferential operators. In Harmonic analysis and applications, Appl. Numer. Harmon. Anal., pages 139-169. Birkhäuser Boston, Boston, MA, 2006.

[13] K. Gröchenig. Time-frequency analysis of Sjöstrand's class. Revista Mat. Iberoam., 22(2):703724, 2006.

[14] K. Gröchenig and Z. Rzeszotnik. Banach algebras of pseudodifferential operators and their almost diagonalization. Ann. Inst. Fourier (Grenoble), 58(7):2279-2314, 2008.

[15] M. Hampejs, P. Svac, G. Tauböck, K. Gröchenig, F. Hlawatsch, and G. Matz. Sequential LSQR-based ICI equalization and decision feedback ISI cancelalation in pulse-shaped multicarrier systems. Proc. IEEE SPAWC09, Helsinki.

[16] L. Hörmander. The analysis of linear partial differential operators. III, volume 274 of Grundlehren der Mathematischen Wissenschaften [Fundamental Principles of Mathematical Sciences]. Springer-Verlag, Berlin, 1994. Pseudo-differential operators, Corrected reprint of the 1985 original.

[17] A. Klotz. Noncommutative approximation: smoothness and approximation and invertibility in Banach algebras. PhD thesis, Univ. Vienna, 2009.

[18] J. Sjöstrand. An algebra of pseudodifferential operators. Math. Res. Lett., 1(2):185-192, 1994.

[19] J. Sjöstrand. Wiener type algebras of pseudodifferential operators. In Séminaire sur les Équations aux Dérivées Partielles, 1994-1995, pages Exp. No. IV, 21. École Polytech., Palaiseau, 1995.

[20] T. Strohmer. Pseudodifferential operators and Banach algebras in mobile communications. Appl. Comput. Harmon. Anal., 20(2):237-249, 2006.

[21] G. Tauböck, M. Hampejs, G. Matz, F. Hlawatsch, and K. Gröchenig. LSQR-based ICI equalization for multicarrier communications in strongly dispersive and highly mobile environments. Proc. IEEE SPAWC07.

[22] J. Toft. Continuity properties in non-commutative convolution algebras, with applications in pseudo-differential calculus. Bull. Sci. Math., 126(2):115-142, 2002.

[23] M. W. Wong. Wavelets Transforms and Localization Operators, volume 136 of Operator Theory Advances and Applications. Birkhauser, 2002.

Faculty of Mathematics, University of Vienna, Nordbergstrasse 15, A-1090 ViENNA, Austria

E-mail address: karlheinz.groechenig@univie.ac.at 